\title{
Insulin Promotes Electrical Coupling between Cultured Sympathetic Neurons ${ }^{1}$
}

\author{
EVE J. WOLINSKY, ${ }^{2}$ PAUL H. PATTERSON, ${ }^{3}$ AND ALAN L. WILLARD ${ }^{4}$ \\ Department of Neurobiology, Harvard Medical School, Boston, Massachusetts 02115
}

\begin{abstract}
Placing neurons in tissue culture is one way to study how environmental factors affect their differentiation. Replacement of serum-supplementation of the culture medium with defined ingredients extends the experimenter's control of the culture environment; however it also introduces additional potential influences. In this report, we confirm the observation of Higgins and Burton (Higgins, D., and H. Burton (1982) Neuroscience 7: 2241-2253) of increased frequency of electrical coupling in serum-free compared to serum-supplemented cultures of rat sympathetic neurons. In addition, experiments were performed to determine whether this effect results from the removal of serum or from the addition of the defined medium components to the culture environment. The results of testing individual ingredients of the defined medium recipe adapted for use on sympathetic neurons (Bottenstein, J. E., and G. H. Sato (1979) Proc. Natl. Acad. Sci. U. S. A. 76: 514-517) show that insulin is capable of inducing electrical coupling in serum-free cultures. Thus, the formation of electrical synapses by sympathetic neurons can be hormonally regulated.
\end{abstract}

One difference between cultures of rat sympathetic neurons grown with or without serum is that serum-free cultures synthesize and store less acetylcholine (lacovitti et al., 1982; Wolinsky, et al., 1985). When the compounds used to replace serum-supplementation in defined medium were combined $v_{\text {, th }}$ serum, no decrease in acetylcholine production was observed (Wolinsky et al., accom-

Received July 2, 1984; Revised October 5, 1984;

Accepted October 8, 1984

${ }^{1}$ This work was supported by grants from the National Institute of Neurological and Communicative Disorders and Stroke (NINCDS). E. J. W. was a predoctoral trainee of the National Institute of General Medical Sciences. P. H. P. was a Mita Allen Foundation Fellow and a McKnight Foundation Neuroscience Development Awardee. A. L. W. was a postdoctoral fellow of the American Heart Association, Massachusetts Affiliate, and is an Alfred P. Sloan Research Fallow. Support was also provided by grants from NINCDS to P. H. P. We would like to thank Doreen McDowell for assistance with the cell culturing. We also thank Drs. S. C. Landis and T. M. Jessell for helpful discussion of the manuscript and Dr. E. J. Furshpan for help with initial experiments

${ }^{2}$ To whom correspondence should be sent, at her present address: Department of Biology, Massachusetts Institute of Technology, Cambridge, MA 02139.

${ }^{3}$ Present address: Division of Biology, California Institute of Technology, Pasadena, CA 91125

${ }^{4}$ Present address: Department of Physiology, University of North Carolina, Medical.Research Building 2064, Chapel Hill, NC 27514. panying manuscript). The implication that the serum is responsible for induction of the cholinergic function was confirmed by the determination of a dose-response relationship between serum concentration and amount of acetylcholine production (Wolinsky and Patterson, 1985). It may therefore be concluded that the lower synthesis and storage of acetylcholine by cultures grown without serum is due to serum having been removed, rather than to inhibitory effects of the compounds replacing it.

A second difference between cultures grown with or without serum is that electrical synapses are common in cultures grown in defined medium (Higgins and Burton, 1982) but rare in cultures grown with serum (Ko et al., 1976; O'Lague, Furshpan, and Potter, 1978). The experiments described in the present report confirm the findings of Higgins and Burton (1982) and test whether the increased frequency of electrical synapses in defined medium cultures is due to removal of serum or to the effects of the compounds used to replace serum. The results indicate that the increased electrical coupling observed under serum-free conditions is due to the presence of insulin-an ingredient of the N2-defined medium recipe described by Bottenstein and Sato (1979) - rather than being due to the removal of serum. Thus, replacement of a complex medium supplement, such as rat serum, with a defined set of hormones and nutrients, can add as well as remove molecules that influence the properties of neurons grown in culture.

Some of the results reported in this paper were previously described in an abstract (Wolinsky, Landis, Patterson, and Willard 1983).

\section{Materials and Methods}

Cell culture. Cultures were prepared from enzymatically dissociated new born rat superior cervical ganglia as previously described (Wolinsky and Patterson, 1983). Cultures maintained in defined medium, N2L 15 or L15 plus insulin and transferrin, were prepared as described by Wolinsky et al. (1985). Cultures maintained in L15 medium plus $5 \%$ rat serum were prepared essentially by the method of Hawrot and Patterson (1979). Rat serum was stored at $-20^{\circ} \mathrm{C}$ which would be expected to inactivate endogenous insulin, which is not stable to freezing.

Electrophysiological techniques. Cultures were placed on the heated stage of a compound microscope, viewed with phase contrast optics, and superfused with a perfusion medium described by O'Lague et al. (1978a). Pairs of neurons (see Fig. 1) were impaled with intracellular microelectrodes filled with $3 \mathrm{M} \mathrm{KCl}$ and having D.C. resistances of 70 to 150 megohms. An active bridge circuit (Getting M-5) permitted the same electrode to be used for both current injection and voltage recording. Coupling ratios were determined by measuring the hyperpolarization caused in one cell when a second cell was injected with sufficient current to cause it to be hyperpolarized by 20 to $50 \mathrm{mV}$. However, the best impalements, as determined by resting and action potential amplitudes and input impedances, were obtained with the higher resistance electrodes. These high resistance electrodes often did not permit unambiguous maintenance of bridge balance, and therefore, in several experiments, qualitative rather than quantitative values were assigned to the strength of the coupling observed between pairs of neurons. The qualitative categories (in declining order of strength) were as follows (illustrated in Fig. 2): (1) suprathreshold ("supra")-eliciting action potentials in one cell caused 

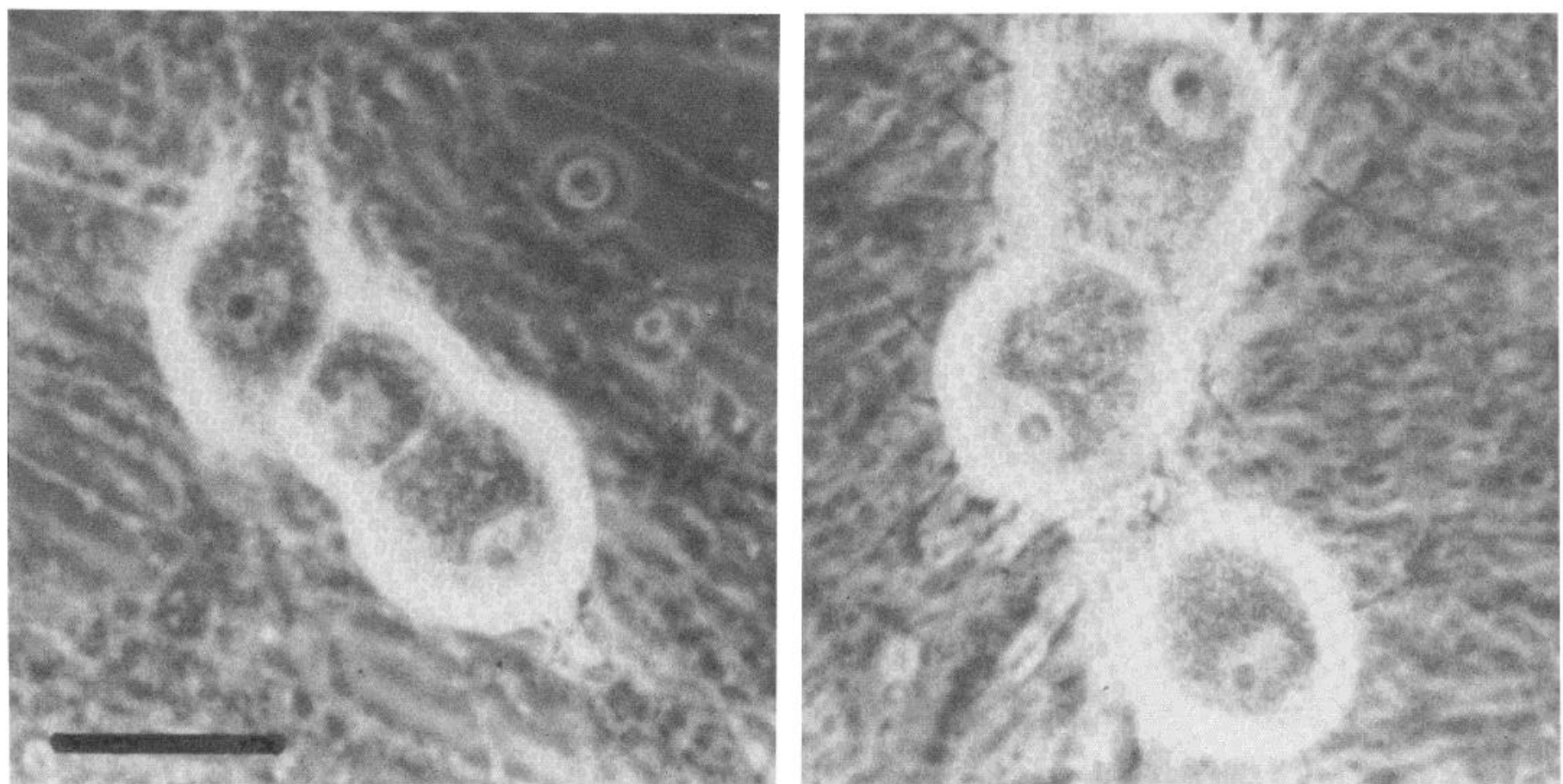

Figure 1. Micrographs of serum-free and serum-containing neurons. The panel on the left shows neurons from a defined-medium culture; the right-hand panel shows neurons from a serum-supplemented culture. All three neurons in the left-hand panel are sufficiently close to their neighbor to be considered "adjacent" for the purpose of selecting pairs to test electrical coupling. In the right-hand panel, only the upper pair of the three was considered to be "adjacent." The bottom-most neuron does not appear to be directly touching its neighbor. The calibration bar indicates a length of $50 \mu \mathrm{m}$.

action potentials in the other cell; (2) "strong"-action potentials in one cell caused subthreshold depolarizing potentials of greater than $2 \mathrm{mV}$ in the other cell; (3) "moderate"-action potentials in one cell caused less than $2 \mathrm{mV}$ depolarizations in the other cell, and hyperpolarizing one cell by 50 to 100 $\mathrm{mV}$ caused a hyperpolarization of more than $2 \mathrm{mV}$ in the other cell; (4) "weak"- depolarizations did not pass between the cells, and very large hyperpolarizations of one cell (100 to $200 \mathrm{mV}$ ) caused less than $2 \mathrm{mV}$ hyperpolarization in the other cell; (5) "undetectable"-no detectable voltage change occurred in one cell, even when the other cell was hyperpolarized by $200 \mathrm{mV}$ or more.

Cultures grown in medium containing rat serum often exhibited high levels of spontaneous chemical (cholinergic) synaptic potentials. These made it difficult to measure coupling and were therefore blocked by adding $1 \mathrm{~mm}$ hexamethonium, a nicotinic ganglionic blocking agent, to the perfusion medium. Hexamethonium had no detectable effect on electrical coupling in either serum-containing or serum-free cultures.

\section{Results}

Pairs of adjacent neuronal cell bodies, as shown in Figure 1, in cultures maintained in growth medium with different combinations of serum and N2L15 components were impaled in their cell bodies and tested for electrical interactions. In both serum-free and serumcontaining cultures, various degrees of electrical coupling were observed. In most experiments, coupling was categorized by the criteria described in "Materials and Methods." These classes are illustrated in Figure 2. In almost all cases, the observed interactions were reciprocal. That is, stimulation of either cell in a pair resulted in the same category of response in the unstimulated partner. Use of the terms pre- and postsynaptic in this context is merely an operational designation of the stimulated and unstimulated members of a pair.

The interactions illustrated in Figure 2 are of very short latency and therefore likely to be electrotonic. Serum-containing cultures have been previously shown to contain some cholinergic synapses (O'Lague et al., 1978a, 1978b). Stimulation-evoked interactions of short and long latency could be clearly distinguished in serumcontaining cultures. Long but not short latency interactions were blocked by $1 \mathrm{~mm}$ hexamethonium. During one recording session, a serum-free culture was perfused with $200 \mu \mathrm{M} \mathrm{CdCl}$ for 20 min. During this time, stimulation-evoked action potentials were of smaller amplitude than in normal perfusion medium. These effects are consistent with the known role of $\mathrm{Ca}^{++}$in impulse propagation (Ko et al., 1976; O'Lague et al., 1978a; Higgins and Burton, 1982) and exocytotic transmitter release in these cultures (Patterson et al., 1975; O'Lague et al., 1978; Buckley and Landis, 1983). However, short-latency, stimulation-evoked interactions persisted in the presence of $\mathrm{Cd}^{++}$. One pair, tested before and during $\mathrm{Cd}^{++}$perfusion, fired action potentials simultaneously during suprathreshold stimulation of one partner, regardless of the presence of $\mathrm{Cd}^{++}$. After a 15-min wash-out, action potential amplitude returned to normal.

The results of a typical experiment comparing electrical coupling in serum-containing and serum-free cultures is shown in Table I. Sister cultures in this plating were tested at 11 and 17 days in vitro. Results obtained during each of these recording sessions were similar. More than half of the pairs tested in serum-free cultures showed strong or suprathreshold coupling, while only $5 \%$ or less of the pairs tested in serum-supplemented cultures exhibited this level of interaction. Coupling was undetectable in the majority of pairs tested in serum-containing cultures, while in no pair tested under serum-free conditions was coupling strength below the level of detection. A third condition was examined in this experiment, the combination of N2 ingredients with rat serum. Addition of N2 ingredients to serum-supplemented medium greatly increased the level of electrical interactions observed. In these cultures, $75 \%$ of the pairs tested showed strong or suprathreshold coupling, and none exhibited electrical interactions below the level of detection. These results are very similar to those obtained under serum-free conditions and are quite different from those with cultures maintained in medium supplemented with serum alone.

To determine whether any individual N2 ingredient could induce coupling, serum-containing medium was prepared to which either insulin $(5 \mu \mathrm{g} / \mathrm{ml})$, transferrin $(100 \mu \mathrm{g} / \mathrm{ml})$, putrescine $(16 \mu \mathrm{g} / \mathrm{ml})$, or selenium $(30 \mathrm{~nm})$ and progesterone $(20 \mathrm{~nm})$ had been added. These 
A
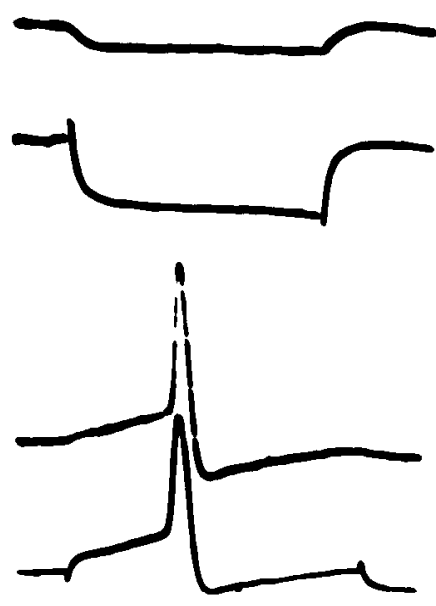

D
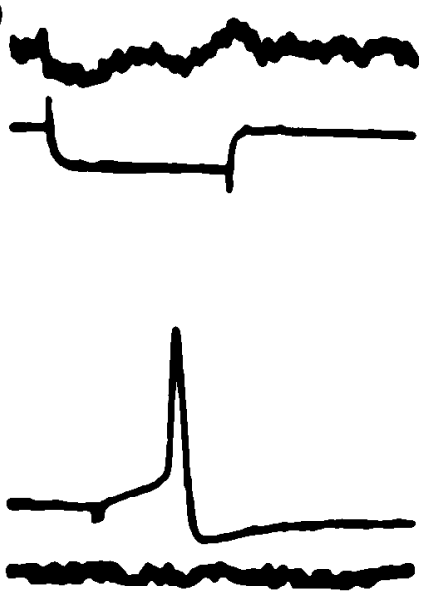
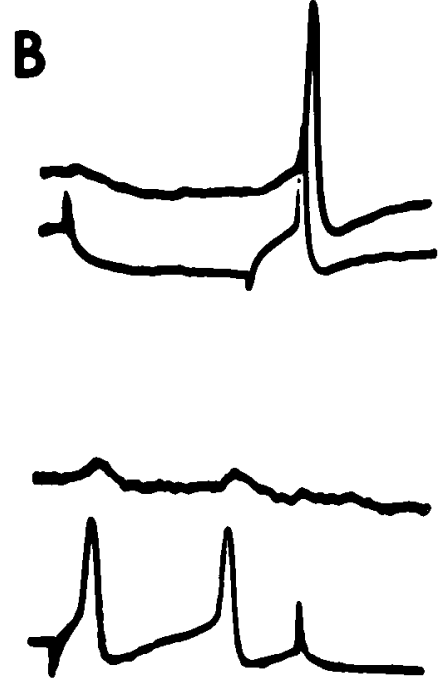

E
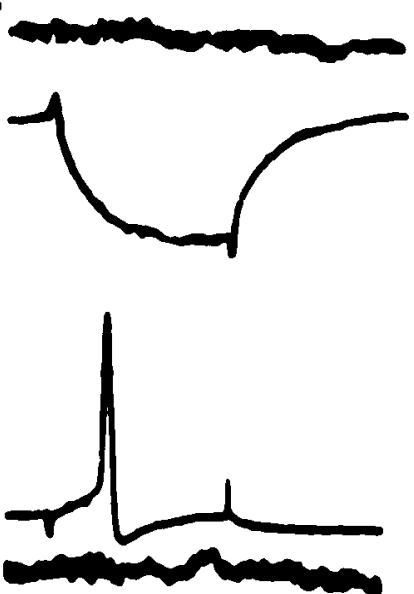
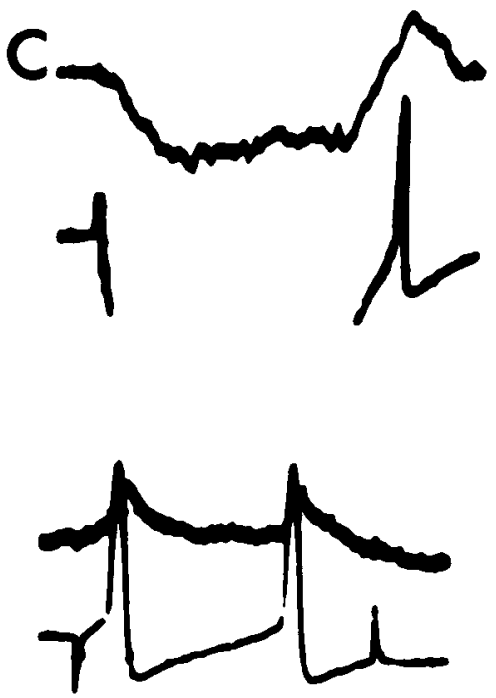

Figure 2. Categories of electrical coupling strength. The upper pair of traces of each panel shows the results of a hyperpolarizing stimulus; the lower pair shows results for depolarization. A, Suprathreshold (all traces: $80 \mathrm{mV}, 20 \mathrm{~ms}$ ); $B$, Strong (upper traces: $80 \mathrm{mV}$; lower traces: $80 \mathrm{mV}$ pre-, $40 \mathrm{mV}$ postsynaptic, all traces $20 \mathrm{~ms}$ ); $C$, Moderate (upper traces: $80 \mathrm{mV}$ pre-, $4 \mathrm{mV}$ postsynaptic, $10 \mathrm{~ms}$; lower traces: same, $20 \mathrm{~ms}$ ); $D$, Weak (upper traces: $400 \mathrm{mV}$ pre-, $2 \mathrm{mV}$ postsynaptic, $40 \mathrm{~ms}$; lower traces: $80 \mathrm{mV}$ pre-, $2 \mathrm{mV}$ postsynaptic, $20 \mathrm{~ms}$ ); $E$, Undetectable (all traces: $80 \mathrm{mV}$ pre-, $2 \mathrm{mV}$ postsynaptic, $20 \mathrm{~ms}$ ).

TABLE I

Effect of defined medium on electrical coupling

Ten or 11 pairs from one dish grown under each of the culture conditions shown were classified by strength of electrical coupling according to the criteria described in the text. The number of pairs falling into each category is shown over the number of pairs tested for each experimental condition. Media used were I 15 supplemented with either rat serum (RS) and/or N2 recipe ingredients (N2).

\begin{tabular}{clccccc}
\hline Age & Medium & ND $^{2}$ & Weak & Moderate & Strong & Supra \\
\hline \multirow{3}{*}{11} & L15N2 & $0 / 10$ & $1 / 10$ & $2 / 10$ & $7 / 10$ & $0 / 10$ \\
& L15RS & $8 / 10$ & $1 / 10$ & $1 / 10$ & $0 / 10$ & $0 / 10$ \\
\multirow{3}{*}{17} & L15N2 + RS & $2 / 10$ & $0 / 10$ & $2 / 10$ & $5 / 10$ & $1 / 10$ \\
& L15N2 & $0 / 11$ & $3 / 11$ & $2 / 11$ & $5 / 11$ & $1 / 11$ \\
& I 15RS & $5 / 10$ & $2 / 10$ & $2 / 10$ & $1 / 10$ & $0 / 10$ \\
& L15N2 + RS & $0 / 10$ & $0 / 10$ & $1 / 10$ & $6 / 10$ & $3 / 10$ \\
\hline
\end{tabular}

${ }^{a} \mathrm{ND}$, not detectable.

separate ingredients were added at the same concentration at which they are present in serum-free L15N2 medium. The results of electrical coupling measurements on cultures fed with these media are shown in Table II. The difference in coupling intensities measured in cultures maintained in L15RS medium and L15N2 medium is even more striking in this plating than in that shown in Table I. These two experiments exhibit the range of frequency of strong and suprathreshold coupling interactions observed in serum-free cultures from several platings. Every pair tested in the experiment shown in Table II in cultures maintained under serum-free conditions exhibited suprathreshold coupling, while $95 \%$ of the coupling observed in L15RS cultures was at the moderate level or weaker. In $35 \%$ of the pairs tested in L15RS cultures, coupling was undetectable. Pairs tested in serum-containing cultures in which transferrin, putrescine, or selenium plus progesterone were present throughout the culture period exhibited no suprathreshold or strong coupling. However, addition of insulin resulted in a $40 \%$ rate of strong and suprathreshold coupling in tested pairs. Although insulin addition to serum-containing medium did not elevate the coupling intensities observed to the same level as in serum-free cultures, this is the only N2 ingredient which changed coupling levels in the presence of serum to this extent. Acute addition of insulin to serum-containing cultures several days prior to electrophysiological experiments did not elevate electrical coupling. Although both putrescine and selenium plus progesterone appear to shift measured coupling intensities from the undetectable and weak to moderate categories, this effect is weaker than that of insulin and was not consistently observed in other platings.

Also shown in Table II are coupling measurements made on pairs 
TABLE ॥

Effect of N2 ingredients on electrical coupling

Ten to 20 pairs from one dish grown under each of seven culture conditions were classified by strength of electrical coupling as described in the text. Data are presented and abbreviations are as in Table I. Concentrations of medium additives tested are reported in the text. intracellular recording was performed on days 20 and 24 in vitro.

\begin{tabular}{lccccc}
\hline \multicolumn{1}{c}{ Medium } & ND $^{2}$ & Weak & Moderate & Strong & Supra \\
\hline L15N2 & $0 / 15$ & $0 / 15$ & $0 / 15$ & $0 / 15$ & $15 / 15$ \\
L15RS & $7 / 20$ & $6 / 20$ & $6 / 20$ & $1 / 20$ & $0 / 20$ \\
L15RS + insulin & $1 / 15$ & $1 / 15$ & $7 / 15$ & $2 / 15$ & $4 / 15$ \\
L15RS + transferrin & $7 / 16$ & $5 / 16$ & $4 / 16$ & $0 / 16$ & $0 / 16$ \\
L15RS + putrescine & $0 / 18$ & $2 / 18$ & $16 / 18$ & $0 / 18$ & $0 / 18$ \\
L15RS + selenium & $1 / 10$ & $0 / 10$ & $8 / 10$ & $0 / 10$ & $1 / 10$ \\
$\quad+$ progesterone & & & & & \\
L15N2K + & $0 / 10$ & $1 / 10$ & $3 / 10$ & $5 / 10$ & $1 / 10$ \\
\hline
\end{tabular}

${ }^{a} \mathrm{ND}$, not detectable

${ }^{b} \mathrm{~K}+$ indicates $\mathrm{L} 15$ medium with $\mathrm{KCl}$ levels elevated to $20 \mathrm{~mm}$.

maintained under serum-free conditions with chronic depolarization. Chronic depolarization was maintained by use of $L 15$ basal medium containing $20 \mathrm{~mm} \mathrm{KCl}$, as described by Walicke et al. (1977) The effect of chronic depolarization on serum-containing cultures is to stabilize the noradrenergic transmitter phenotype expressed by neonatal rat superior cervical ganglion neurons (evidence summarized by Landis, 1980) against the cholincrgic influcnce of adult rat serum (Wolinsky and Patterson, 1985). Does this phenotypic stabilization by chronic depolarization extend to the membrane properties of the neurons? In $60 \%$ of the pairs tested, coupling was in the strong or suprathreshold category, despite chronic depolarization during the culture period prior to the recording session. Although this rate is not as high as in the pairs tested in nondepolarized, serum-free sister cultures, it is higher than that of the tested pairs from L15RS cultures.

A more quantitative assessment of the influence of insulin on electrical coupling was made using recording techniques which produced a numerical measure of coupling strength based on the ratio of membrane potentials recorded in pairs during hyperpolarization of one partner. Io avoid variability due to possible slight effects of putrescine, progesterone, and selenium, a further simplified serum-free culture medium was employed containing only two of the N2 recipe ingredients, insulin and transferrin. This simplified medium has been previously shown to support the growth and differentiation of sympathetic neurons in culture (Wolinsky et al., 1985). Numerical coupling measurements made in cultures maintained in L15 medium supplemented with either rat serum, insulin plus transferrin, or rat serum plus insulin are shown as histograms in Figure 3. Figure 3 a shows that the population of observed coupling ratios in L15RS and L15 serum-free (insulin and transferrin supplementation) cultures are distinct and nonoverlapping. The means of the determined coupling ratios differ by five-fold, which is significant by the criterion of two-tailed $t$-testing. In Figure $3 b$, results obtained from cultures supplemented with both serum and insulin are shown. This distribution is broader than and overlaps the other two. Its mean, although intermediate between the other two, is significantly different from each by two-fold.

\section{Discussion}

The results reported here show that insulin is capable of inducing electrical coupling in cultured sympathetic neurons. This conclusion provides an explanation for the higher levels of electrical coupling observed in serum-free than in serum-supplemented cultures, since insulin is an essential component of the defined medium. The types of electrical coupling reported here are qualitatively similar to those previously reported by Higgins and Burton (1982) in serum-free sympathetic neurons cultures using slightly different techniques for culturing and intracellular recording.
The conclusion that insulin promotes formation of electrical synapses between cultured sympathetic neurons is based on frequency with which electrical interactions of various strengths were observed in test populations of adjacent pairs. The assumption has been made that the observed coupling interactions in the test samples (10 to 20 pairs per experiment per variable) reflect the overall distribution of coupling strengths in the entire culture population (1000 to 3000 neurons per dish). This is subject to the reservation that the sampled population may have been biased according to suitability for obtaining reliable intracellular recordings: features such as size, high input resistance after impalement, and stability of responses. Such considerations may be most important in comparison of serum-free and serum-containing cultures, in which the presence or absence of serum may be influential, as well as that of insulin. For example, cell body diameter appears to be smaller and membrane fragility greater in serum-free than in serum-containing cultures (nonquantitative observations). However, comparison of serum-containing cultures with and without insulin, as shown in Figure 3 , isolates the presence of insulin as a single variable and results in the same conclusion that insulin induces electrical coupling. Conclusions regarding the ability of other N2 ingredients to stimulate electrical coupling cannot be made from the data presented here, other than that any effect they might have is much weaker than that of insulin and would not be reliably detected in our experiments.

The effect of insulin is not abolished by chronic depolarization, which promotes maintenance of the noradrenergic phenotype. Thus, stabilizalion of transmitter phenolype does not prevent induction of electrical coupling. Further evidence that coupling is not restricted by transmitter choice is that the degree of electrical coupling exhibited by a particular neuron pair does not seem to depend on the transmitter properties of the presynaptic cell. Serum-free cultures are essentially noradrenergic (Wolinsky et al., 1985), and it is in these cultures that strong electrical interactions were most frequently observed. However, in serum-containing cultures, which consist of a neuronal population mixed with respect to transmitter phenotype, some pairs tested exhibited both short and long latency interactions. The long latency interactions were typical of cholinergic excitatory postsynaptic potentials. Thus, electrical transmission can coexist with cholinergic transmission in cultured sympathetic neurons.

The significance of electrical synapses in sympathetic neurons is not clear. Coupling has not been reported between principal neurons of the adult superior cervical ganglion, and whether coupling exists transiently in early development of the sympathetic nervous system is not known. However, ciliary neurons of the chick ciliary ganglion participate in electrical synapses with preganglionic neurons (Martin and Pilar 1963, 1964; Landmesser and Pilar, 1972). Both sympathetic and parasympathetic neurons originate in the neural crest during development, and it is possible that electrical transmission is part of the environmentally controlled phenotypic repertoire available to neural crest derivatives that is not elicited during normal development of sympathetic neurons. The role of insulin as an inducer of this trait in culture does not have a clear significance. It is possible that a contaminant of the insulin preparation employed is responsible rather than insulin itself, since the dose used $(5 \mu \mathrm{g} / \mathrm{ml})$ is unphysiologically high. However, a serum-free culture maintained in a 100-fold lower concentration of insulin also exhibited coupling levels elevated over non-insulin-supplemented, serum-containing sister cultures (data not shown). It is also possible that insulin is acting via receptors with higher affinity for a different molecule, as suggested by Barnes and Sato (1980).

The anatomical basis of electrical coupling in a number of tissues is considered to be the gap junction (Bennett and Goodenough, 1978). Experiments addressing the question of whether the physiologically measured coupling reported here is related to gap junctional connections between cells have not been done. However, the electrophysiological characteristics of the coupling observed in sympathetic neuron cultures are not incompatible with transmission via gap junctions. Factors regulating formation of electrically conducting 


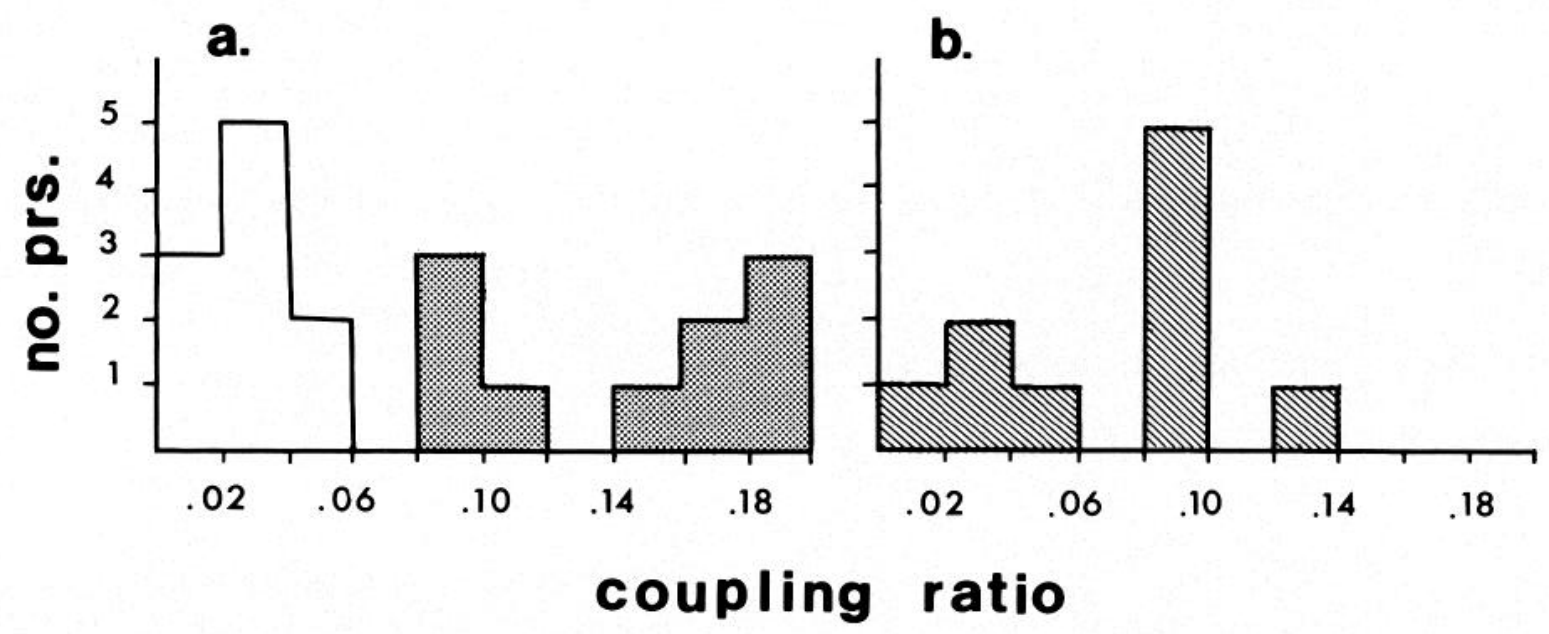

Figure 3. Effect of insulin on distribution of coupling intensity. The histograms show the number of pairs tested the coupling ratios of which fell within the indicated bins. The first bin included ratios less than 0.02 but includes no measurements lower than 0.005 . Ten pairs were tested for each of the indicated culture conditions on day 27 in vitro. The clear, stippled, and hatched bars represent, respectively, results from cultures grown with serum, insulin and transferrin without serum, and serum plus insulin. Means \pm SE of coupling ratios for each experimental condition are: $0.030 \pm 0.005$ for cultures grown with serum; $0.153 \pm 0.14$ for cultures grown with insulin and transferrin without serum; $0.076 \pm 0.012$ for cultures grown with serum plus insulin. These means are significantly different, with $p<0.001$ by a two-tailed $t$-test for the serum alone compared to serum-free conditions, $p<0.01$ for serum compared to serum plus insulin, and $p<0.001$ for serum-free compared to serum plus insulin cultures.

junctions in characteristic tissues are not currently understood. The role of insulin in promoting formation of electrically conducting, lowresistance connections between cultured sympathetic neurons suggests the possibility of hormonal regulation of this process.

Note: During preparation of this manuscript, we learned that others have also found that insulin promotes electrical coupling in sympathetic neurons. These investigators also report a larger effect of selenium in promoting electrical coupling than we have observed (Kessler et al., 1984).

\section{References}

Barnes, D., and G. H. Sato (1980) Serum-free cell culture: A unifying approach. Cell 22: 649-655.

Bennett, M. V., and D. A. Goodenough (1978) Gap junctions, electrotonic coupling, and intercellular communication. Neurosci. Res. Program Bull. 16 (3).

Bottenstein, J. E., and G. H. Sato (1979) Growth of a rat neuroblastoma line in serum-free supplemented medium. Proc. Natl. Acad. Sci. U. S. A. 76: 514-517.

Buckley, K. M., and S. C. Landis (1983) Morphological studies of neurotransmitter release and membrane recycling in sympathetic nerve terminals in culture. J. Neurocytol. 12: 93-116.

Hawrot, E., and P. H. Patterson (1979) Long-term culture of dissociated sympathetic neurons. Methods Enzymol. 58: 574-584.

Higgins, D., and H. Burton (1982) Electrotonic synapses are formed by fetal rat sympathetic neurons maintained in a chemically defined medium. Neuroscience 7: 2241-2253.

lacovitti, L., M. I. Johnson, T. H. Joh, and R. P. Bunge (1982) Biochemical and morphological characterization of sympathetic neurons grown in a chemically defined medium. Neuroscience 7: 2225-2239.

Kessler, J. A., D. C. Spray, J. C. Saez, and M. V. Bennett (1984) Determination of synaptic phenotype: Insulin and cAMP independently initiate develop- ment of electrotonic coupling between cultured sympathetic neurons. Proc. Natl. Acad. Sci. U. S. A. 81: 6235-6239.

Ko, C. -P., H. Burton, M. I. Johnson, and R. P. Bunge (1976) Synaptic transmission between rat superior cervical ganglion neurons in dissociated cell cultures. Brain Res. 117: 461-485.

Landis, S. C. (1980) Developmental changes in the neurotransmitter properties of dissociated sympathetic neurons: A cytochemical study of the effects of medium. Dev. Biol. 77: 349-361.

Landmesser, L. and G. Pilar (1972) The onset and development of transmission in the chick ciliary ganglion. J. Physiol. 222: 691-713.

Martin, A. R., and G. Pilar (1963) Dual mode of synaptic transmission in the avian ciliary ganglion. J. Physiol. 168: 443-463.

Martin, A. R. and G. Pilar (1964) An analysis of electrical coupling at synapses in the avian ciliary ganglion. J. Physiol. 171: 454-475.

O'Lague, P. H., E. J. Furshpan, and D. D. Potter (1978) Studies on rat sympathetic neurons developing in cell culture. II. Synaptic mechanisms. Dev. Biol. 67: 404-423.

O'Lague, P. H., D. D. Potter, and E. J. Furshpan (1978a) Studies on rat sympathetic neurons developing in cell culture. I. Growth characteristics and electrophysiological properties. Dev. Biol. 68: 384-403.

O'Lague, P. H., D. D. Potter, and E. J. Furshpan (1978b) Studies on rat sympathetic neurons developing in cell culture. III. Cholinergic transmission. Dev. Biol. 68: 424-444.

Patterson, P. H., L. F. Reichardt, and L. L. Y. Chun (1976) Biochemical studies on the development of primary sympathetic neurons in cell culture. Cold Spring Harbor Symp. Quant. Biol. 40: 389-397.

Walicke, P. A., R. B. Campenot, and P. H. Patterson (1977) Determination of transmitter function by neuronal activity. Proc. Natl. Acad. Sci. U. S. A. 74: $5767-5771$.

Wolinsky, E. J., and P. H. Patterson (1985) Rat serum contains a developmentally regulated cholinergic inducing activity. J. Neurosci. 5: 1509-1512.

Wolinsky, E., S. C. Landis, P. H. Patterson, and A. Willard (1983) Influence of serum on cultured sympathetic neurons. Soc. Neurosci. Abstr. 9: 4.

Wolinsky, E. J., S. C. Landis, and P. H. Patterson (1985) Expression of noradrenergic and cholinergic traits by sympathetic neurons cultured without serum. J. Neurosci. 5: 1497-1508. 